\title{
Specific lipid lowering therapy in the management of diabetes
}

\author{
P.N. Durrington
}

University of Manchester Department of Medicine, Manchester Royal Infirmary, Oxford Road, Manchester M13 9WL, UK

\section{Introduction}

Doctors visiting the UK from overseas are frequently amazed that there are still physicians here who seem to think that whether to treat hyperlipidaemia to prevent coronary heart disease (CHD) is a matter for debate, when in reality the evidence favouring such an approach is often stronger than that for many other disorders, which are treated without question. ${ }^{1}$ Certainly there are grounds to debate the most appropriate level of cholesterol at which drug treatment should be instituted in different groups of patients, ${ }^{2-4}$ but that certain patients stand to benefit greatly from treatment for hyperlipidaemia can no longer be denied. The evidence for this has recently been reviewed in detail elsewhere ${ }^{1,5,6}$ and I shall therefore here simply review only that which is of relevance to the management of hyperlipidaemia as a means of coronary prevention in diabetes mellitus in clinical practice. Details of lipoprotein physiology and other information not included in the present article can be found in two earlier articles. ${ }^{7,8}$

\section{Lipoproteins as risk factors for atherosclerosis in diabetes}

The relationship between serum cholesterol and the risk of CHD was first clearly established in the Framingham study ${ }^{9}$ and has since been irrefutably confirmed. ${ }^{10}$ Serum cholesterol in diabetes, as in the general population, is related to CHD risk, the only difference being that the risk rises even more steeply with increasing cholesterol. ${ }^{11}$ In the general population the risk from cholesterol is largely attributable to the low density lipoprotein (LDL) cholesterol. The other major component of the total serum cholesterol, high density lipoprotein (HDL) cholesterol, is inversely related to the risk in most studies. ${ }^{12}$ In diabetes the same relationship between LDL cholesterol holds true, but in addition a greater proportion of the serum cholesterol is

Correspondence: P.N. Durrington, M.D., F.R.C.P. present in very low density lipoprotein (VLDL) and intermediate density lipoprotein (IDL). There is accumulating evidence to link VLDL and IDL with accelerated atherogenesis. ${ }^{13,14}$ Since these lipoproteins are rich in triglycerides as well as cholesterol, this may explain the much stronger relationship between CHD risk and serum triglycerides in diabetes compared to the general population. . $^{13,15}$

There is evidence that in non-insulin dependent diabetes mellitus (NIDDM), as in the general population, serum HDL levels are inversely related to risk. ${ }^{11}$ The confusing factor in insulin-dependent diabetes mellitus (IDDM) is that either the disease itself or the insulin treatment has set the average HDL cholesterol level above that of the non-diabetic population. ${ }^{7,13}$ Even so, there is some evidence that within the IDDM population HDL is still inversely related to the risk of arterial disease. ${ }^{16}$ In NIDDM the factors regulating HDL levels, for example obesity and hypertriglyceridaemia appear to be the same as in the non-diabetic population. ${ }^{17}$

\section{Clinical trials relevant to diabetic practice}

Two important primary prevention studies in middle-aged men with no preceeding evidence of CHD, the Lipid Research Clinics (LRC) Study ${ }^{18,19}$ and the Helsinki Heart Study, ${ }^{20,21}$ have proved that lowering serum cholesterol decreases coronary morbidity and mortality. In neither trial were the serum cholesterol levels before starting drug therapy extremely high, being 7.2 in the LRC trial and $6.9 \mathrm{mmol} / \mathrm{l}$ in the Helsinki Heart Study. Furthermore, men with evidence of pre-existing CHD at the outset were rigorously excluded. In neither of these trials was there a decrease in overall death rates. This was because the men participating in the study were so healthy at the outset that coronary death rates were not high and did not assume a great enough proportion of total deaths to make a significant impact on overall mortality, which, as is the case in middle-aged men whether they are in a 
trial or not, was due to malignancy and accidents as well as CHD. These trials thus tell us two things. Firstly, that by decreasing cholesterol we can reduce coronary morbidity and CHD deaths, but secondly, that if we want to have any worthwhile impact in extending life we had better turn to a population whose chance of premature death due to CHD is much greater than from other causes. Thus, for example, in the Stockholm Secondary Prevention study ${ }^{22}$ in which myocardial infarction survivors (whose chances of dying of a cause, other than CHD, are much less than the general population) were randomized to receive cholesterol-lowering medication or placebo, there was a decrease in both coronary (36\%) and total mortality (26\%) over 10 years.

Diabetic patients, many of whom will have hyperlipidaemia and other risk factors for CHD, are at greatly increased risk of dying prematurely of CHD and we do not have to be greatly gifted as clinicians to identify some individuals virtually certain to do so. Thus coronary prevention in diabetes by lipid-lowering is justified by present evidence.

In the LRC trial, which employed cholestyramine as the active drug, a $1 \%$ decrease in cholesterol was associated with a $2 \%$ decrease in CHD morbidity and mortality, ${ }^{19}$ whereas in the Helsinki Heart Study a similar decrease in cholesterol produced a $3 \%$ decrease in CHD. ${ }^{20}$ In the latter study, which was of very similar design to the LRC study (each had about 4,000 men randomly assigned to placebo or active drug), gemfibrozil was the active drug. This had the additional effect of lowering triglycerides and raising HDL and it is likely that one or both of these effects explains its greater efficiency in decreasing CHD. ${ }^{21}$ Of particular importance to the diabetologist, it was patients with type IIb hyperlipoproteinaemia (combined hypercholesterolaemia and hypertriglyceridaemia) who showed the greatest decline in CHD and this combined type of hyperlipidaemia is prevalent in both IDDM and NIDDM. . $^{23,24}$

Another important lession from the LRC and Helsinki Heart trial is the rapidity with which the decline in CHD occurred, the difference between placebo and active therapy being evident after only 2 years in both. A similar rapid decline occurred in the UK in both CHD deaths and diabetes-related deaths during World War II, probably associated with a dramatic decrease in our national fat consumption and an increase in carbohydrate intake in the form of less highly refined flour. ${ }^{25}$

\section{Screening the diabetic clinic}

It is sad to note that, at the present time, most hyperlipidaemia in diabetes is being discovered by the general practitioner. Failure to institute a programme of screening for hyperlipidaemia in the diabetic clinic itself, one of the main purposes of which should be the early detection of complications and their precedents, can no longer have any rational basis. It can only stem from a lack of knowledge on the part of diabetic specialists, recalcitrance due to the feeling that they already have too much to do, or the incorrect belief that it would be expensive or that the patient must be fasting (which poses particular problems for the diabetic). CHD is the commonest cause of serious morbidity and premature death in diabetes. A service which recognizes only other complications must be viewed as a failure. The cost of measuring serum cholesterol is only fractionally more than blood glucose and substantially less than a biochemical profile or a blood count. Fasting has no significant effect on the level of cholesterol in the vast majority of patients. Screening for hypercholesterolaemia can thus be reasonably undertaken using random samples. Our best evidence for benefit of treatment is for cholesterol so that this would be a very reasonable starting point for most diabetic clinics. Several authorities, including $\mathrm{Dr}$ R.S. Elkeles (personal communication, 1990) also advocate the measurement of triglycerides randomly, since, although they will increase following meals, the use of higher levels to define the upper limit of normal than are used in the fasting state (say $4 \mathrm{mmol} / \mathrm{l}$ as opposed to $2 \mathrm{mmol} / \mathrm{l}$ ) would at least allow the detection of those patients with markedly elevated levels, in whom treatment might be important.

Current evidence indicates that we should be focussing our attention on patients whose serum cholesterol exceeds $6.5 \mathrm{mmol} / \mathrm{l}^{4,26}$ (Table I). After attention to diet and to diabetic control and revision of any other therapy, such as antihypertensive therapy, which might elevate lipids, some patients will remain in whom specific lipid-lowering drug therapy may be considered and it is in this group that fasting-cholesterol, triglyceride and HDL-cholesterol may be helpfully determined.

\section{The diabetic diet and hyperlipidaemia}

The chosen diet of diabetic patients in England, even prior to the onset of diabetes, is rich in fat $^{27}$ and it was clearly demonstrated in the 1930s that glucose tolerance deteriorates further when a diet restricted in carbohydrate is given. ${ }^{28}$ This was largely forgotten until recent years and the usual diet for the patient with NIDDM was one of carbohydrate restriction, all the more regrettable since the patient may well have increased fat intake to compensate for the loss of dietary energy in carbohydrate. Furthermore, carbohydrate restric- 
Table I The approach to the managment of the diabetic patient whose serum cholesterol exceeds $6.5 \mathrm{mmol} / 1$

1 a) Dietician (to ensure patient has body weight control and reduction in fat intake as the primary objective of their diet)

b) Smoking (advise to stop in no uncertain terms)

c) Optimise glycaemic control (discuss with dietician: may be better to decrease insulin therapy in order to allow obese patient to lose weight)

2 Repeat measurement of cholesterol. If level still exceeds $6.5 \mathrm{mmol} / \mathrm{l}:-$

3 a) Adequately exclude secondary causes other than diabetes, particularly alcohol abuse, hypothyroidism, nephropathy, which may require therapy in their own right.

b) Consider change of diabetic therapy

i) IDDM - further adjustment of insulin dose/ frequency

ii) NIDDM on diet alone, who is hyperglycaemic consider metformin* if obese, or introduction of sulphonylurea or insulin if underweight

iii) NIDDM on sulphonylurea - consider change to metformin or addition of metformin* depending on blood sugar

c) Does the patient already have CHD? Consider aspirin and further cardiac investigation (exercise ECG and/or coronary angiography)

d) Consider whether any diuretic or $\beta$ adrenoreceptor blocking therapy could be stopped or replaced with another drug.

4 Repeat measurement of cholesterol. If level still exceeds $6.5 \mathrm{mmol} / \mathrm{l}:-$

$5 \quad$ Consider specific lipid-lowering drug

*The occasional patient will tolerate and respond to guar gum.

tion is a very poor means of weight reduction in the obese. The correct diabetic diet is one in which fat is restricted. In the obese diabetic, fat intake should be reduced to achieve a total dietary energy input of 1,000 Cals and the patient should be clear that weight reduction is their primary objective. In the non-obese, fat, particularly saturated fat, should be avoided and the energy deficit bridged with unrefined carbohydrate foods (bread, potatoes, pasta, rice, pulses and beans) and small quantities of polyunsaturated oils (sunflower, corn, safflower, soya bean) or, perhaps preferably, if the patient can afford it, the largely monounsaturated olive oil..$^{29}$ Although this type of dietary approach has been recommended by the British Diabetic Association for several years, too many patients still believe they should avoid carbohydrates and dieticians frequently place too much emphasis on increasing fibre intake as an end in itself.

\section{Diabetic therapy and hyperlipidaemia}

The drugs metformin and guar gum probably have some small direct effect in improving serum lipids not possessed by sulphonylureas, ${ }^{30,31}$ as discussed by Elkeles (this issue, pp. 931-937). Insulin too has the effect of lowering triglycerides and sometimes cholesterol $^{31-33}$ The apparently beneficial effect of insulin and the neutral effect of sulphonylureas may not be borne out in practice and even lead to deterioriation, if their prescription is associated with weight gain. Avoidance of obesity is of the greatest importance in the management of hyperlipidaemia.

\section{Other drugs likely to adversely effect serum lipids}

The effects of diuretic and $\beta$-blocker drugs ${ }^{13,31}$ have already been discussed (Feher, this issue, pp. 938-946). Clearly, for heart failure or some patients with angina, there may be an absolute indication for such therapies, but often discontinuing such treatment or replacing it with other drugs can reasonably be undertaken. This may be particularly important in the management of hypertension in the type of patient who falls into the category of chronic cardiovascular risk discussed by Dodson and Bain (this issue, pp. 922-927).

\section{Specific lipid lowering drug therapy}

\section{Fibric acid derivatives}

This group of drugs includes bezafibrate, fenofibrate and gemfibrozil. The effect of these drugs is principally to decrease serum triglycerides by decreasing VLDL. ${ }^{34}$ In addition they reduce the serum cholesterol concentration particularly when it is raised in association with increased serum triglycerides. The decrease in cholesterol is mainly due to a reduction in the circulating levels of the more lipid-rich LDL subfractions or IDL, ${ }^{35}$ which are the lipoproteins which may be most closely associated with atheroma in diabetes. In addition, there is often a tendency for HDL cholesterol levels to increase during treatment with these drugs. Furthermore, there may be small improvements in glucose tolerance associated with their use. ${ }^{36}$ Bezafibrate has the additional potential advantage that it lowers serum fibrinogen,${ }^{37}$ which contributes to the risk of arteriosclerosis in diabetes. ${ }^{38}$ Gemfibrozil, on the other hand, has the advantage that it has been clearly documented to reduce coronary morbidity and mortality in the Helsinki Heart Study, as previously discussed. The choice of fibrate depends on the individual clinician's judgement and patient acceptability. Generally this group of drugs is well tolerated, and when one of them is not, it is often worth trying one of the others. They should all be avoided in renal insufficiency or gross proteinuria, but, when serum 
creatinine is not elevated and there is only microalbuminuria or macroalbuminuria of less than $1 \mathrm{~g} /$ day, they may be used. Indeed, patients with even minor degrees of nephropathy may be at considerably increased risk of premature CHD and are a group in whom lipid-lowering therapy may be especially important. ${ }^{39,40}$

Fibrates are first-line agents in diabetic patients with an increase in cholesterol associated with hypertriglyceridaemia. ${ }^{34,36,41,42}$ I am not, at the present time, a strong advocate of treating hypertriglyceridaemia when the serum cholesterol is less than $6-6.5 \mathrm{mmol} / \mathrm{l}$. The fibrates would not be suitable drugs for those adventurous enough to do so, because, although they still have the effect of lowering the triglycerides when the LDL cholesterol is low, they tend to increase the more dense LDL, ${ }^{35,43}$ which may counteract any potentially favourable effect.

\section{HMG-CoA reductase inhibitors}

Inhibitors of the enzyme, 3-hydroxy 3-methyl glutaryl-CoA reductase, which include the drugs pravastatin and simvastatin, are relatively new in clinical practice. ${ }^{34}$ Their predominant effect is to decrease LDL, both IDL and the more dense LDL subfractions. They have a relatively small effect in decreasing serum triglycerides compared to the fibrate drugs. They do, however, generally reduce serum LDL cholesterol levels in hypertriglyceridaemia even when the total cholesterol concentration is less than $6.5 \mathrm{mmol} / 1$. They may therefore have a special role in patients, who have established CHD, such as those who have undergone coronary artery surgery in whom it is considered desirable to aim for levels of cholesterol of less than $6 \mathrm{mmol} / \mathrm{l}$. They are thus the treatment of choice in diabetic patients, who have not responded well to fibrate drugs or whose initial serum cholesterol level is substantially elevated, but whose serum triglycerides are not grossly raised, and in those patients in whom particularly low levels of serum cholesterol of less than $6 \mathrm{mmol} / \mathrm{l}$ are regarded as a therapeutic target. They should not generally be used in combination with fibrate drugs as the combination is more likely to produce myositis. Because of the possibility of such a side-effect, although rare when they are used alone, it is recommended that patients have their serum creatine kinase monitored after their introduction and it should, of course, be determined should any muscle symptoms develop. The statins are generally well tolerated, are effective in diabetes and do not cause any deterioriation in glycaemic control. ${ }^{42,44,45}$ They may be used in patients with renal disease, which is another potential advantage in diabetes. ${ }^{46-48}$

\section{Other lipid lowering drugs}

The bile-acid sequestrating agents (cholestyramine and colestipol) are effective cholesterol-lowering drugs. In diabetes, however, they have certain disadvantages. Firstly they tend to increase serum triglycerides ${ }^{49}$ and in diabetes for reasons already discussed this may be more unfavourable and more marked than in primary hyperlipidaemia. Secondly, their mode of administration may make them unacceptable to patients already on multiple medication.

Acipimox is an analogue of nicotinic acid which may be of potential in diabetes. Nicotinic acid itself, although an effective agent in lowering both cholesterol and triglycerides, causes flushing, which is unacceptable to most non-diabetic patients and certainly is to diabetics, who may flush even more. Also, its effect in lowering circulating non-esterified fatty acids (NEFA), which should be an advantage to the diabetic, in whom these contribute to insulin resistance, is only short-lived and is followed by a rebound during which hyperglycaemia may occur. The flushing is less with acipimox and the favourable effect of suppressing serum NEFA may be translated into improved glycaemic control, if sufficiently long-acting formulations are developed. ${ }^{50}$

Fish oil, although it lowers serum triglycerides, often raises the LDL cholesterol and may cause a deterioration in glycaemic control. ${ }^{51-53}$ Probucol, although it may have favourable potential, because it is an antioxidant, lowers serum HDL and it has not been satisfactorily established that it does not have deleterious effects on reverse cholesterol transport. $^{54}$

\section{Conclusion}

There are patients with diabetes at considerable risk of CHD at an early age, many of whom already attend diabetic clinics, many of whom can be easily identified and who have the type of hyperlipidaemia, which it is known can be effectively and beneficially treated. So far as these individuals are concerned there can be no case for further delay.

\section{Acknowledgements}

I should like to thank Miss C. Price for expertly typing this manuscript. 
1. Steinberg, D. The cholesterol controversy is over. Why did it take so long? Circulation 1989, 80: 1070-1078.

2. The Expert Panel. Report of the National Cholesterol Education Program Expert Panel on detection, evaluation and treatment of high blood cholesterol in adults. Arch Intern Med 1988, 148: 36-39.

3. Study Group of the European Atherosclerosis Society. The recognition and management of hyperlipidaemia in adults. A policy statement of the European Atherosclerosis Society. Eur Heart J 1988, 9: 571-600.

4. Shepherd, J., Betteridge, D.J., Durrington, P.N. et al. Strategies for reducing coronary heart disease and desirable limits for blood lipid concentrations: guidelines from the British Hyperlipidaemic Association. Br Med J 1987, 295: 1245-1246.

5. Thompson, G.R. Evidence that lowering serum lipids favourably influences coronary heart disease. $Q J \mathrm{Med} 1987$, 238: $87-95$.

6. Durrington, P.N. Hyperlipidaemia: Should we treat patients? Should we treat populations? What treatment should we use? Recent Adv Cardiol 1992, 11 (in press).

7. Durrington, P.N. \& Winocour, P.H. Therapeutic aspects of hyperlipidaemia in diabetes. Postgrad Med J 1989, 65 (suppl 1): 533-541.

8. Bhatnagar, D. \& Durrington, P.N. Borderline hypercholesterolaemia: when to introduce drugs. Postgrad Med J 1989, 65: $543-552$.

9. Dawber, T.R. The Framingham Study: the epidemiology of atherosclerotic disease. Harvard University Press, Cambridge, Massachusetts 1980.

10. Martin, M.H., Hulley, S.B., Browner, W.S. et al. Serum cholesterol, blood pressure and mortality: implications from a cohort of 361,662 men. Lancet 1989, ii: 933-936.

11. Kannel, W.B. \& McGee, D.L. Diabetes and cardiovascular risk factors: the Framingham Study. Circulation 1979, 59: $8-13$.

12. Gordon, D.J. \& Rifkind, B.M. High density lipoprotein. The clinical implications of recent studies. N Engl J Med 1989, 321: $1311-1316$

13. Durrington, P.N. Secondary hyperlipidaemia. Br Med Bull 1990, 46: 1005-1024.

14. Fontbonne, A., Eschwege, E., Cambien, F. et al. Hypertriglyceridaemia as a risk factor of coronary heart disease mortality in subjects with impaired glucose tolerance or diabetes: results from the 11-year follow-up of the Paris prospective study. Diabetologia 1989, 32: 300-304.

15. West, K.M., Ahuja, M.M., Bennett, P.H. et al. The role of circulating glucose and triglyceride concentrations and their interactions with other 'risk factors' as determinants of arterial disease in nine diabetic population samples from the WHO Multinational Study. Diabetes Care 1983, 6: 361 - 369.

16. Reckless, J.P.D., Betteridge, D.J., Wu, P., Payne, B. \& Galton, D. High-density and low-density lipoproteins and prevalence of vascular disease in diabetes mellitus. $\mathrm{Br} \mathrm{Med} \mathrm{J}$ 1978, 1: 883-886.

17. Durrington, P.N. Serum high density lipoprotein cholesterol in diabetes mellitus: an analysis of factors which influence its concentration. Clin Chim Acta 1980, 104: 11-23.

18. Lipid Research Clinics Program. The Lipid Research Clinics Coronary Primary Prevention Trial results. I. Reduction in incidence of coronary heart disease. JAMA 1984, 251: 351-364.

19. Lipid Research Clinics Program. The Lipid Research Clinics Program. The Lipid Research Clinics Coronary Primary Prevention Trial results. II. The relationship of reduction in incidence of coronary heart disease to cholesterol lowering. JAMA 1984, 251: 365-374.
20. Frick, M.H., Elo, O., Haapa, K. et al. Helsinki Heart Study: primary prevention trial with gemfibrozil in middle-aged men with dyslipidaemia. $N$ Engl J Med 1987, 317: 1237-1245.

21. Manninen, V., Elo, O., Frick, M.H. et aI. Lipid alterations and decline in the incidence of coronary heart disease in the Helsinki Heart Study. JAMA 1988, 260: 641-651.

22. Carlson, L.A. \& Rosenhamer, G. Reduction of mortality in the Stockholm Ischaemic Heart Disease Secondary Prevention Study by combined treatment with alofibrate and nicotinic acid. Acta Med Scand 1988, 223: 405-418.

23. Winocour, P.H., Durrington, P.N., Ishola, M., Hillier, V.F. \& Anderson, D.C. The prevalence of hyperlipidaemia and related clinical features in insulin-dependent diabetes mellitus. $Q J$ Med 1989, 70: 265-276.

24. Taskinen, M.-R., Kuusi, T. \& Nikkila, E.A. Serum lipoproteins and atherosclerosis in type 1 and type 2 diabetes. In Andrean, D., Crepaldi, G., Di Mario, U. \& Pozza, G. (eds) Diabetic Complications: Early Diagnosis and Treatment. 1987, John Wiley \& Sons, New York, 1985, Chapter 3, pp. 25-35.

25. Trowell, H. Diabetes mellitus death-rates in England and Wales 1920-1970 and food supplies. Lancet 1974, ii: 998-1001.

26. Durrington, P.N. Normal serum lipid and lipoprotein concentrations. In: Hyperlipidaemia. Diagnosis and Management. Wright, London, 1989, pp. 56-86.

27. Himsworth, H.P. \& Marshall, E.M. The diet of diabetics prior to the onset of disease. Clin Sci 1935, 2: 95-115.

28. Himsworth, H.P. The dietetic factor determining the glucose tolerance and sensitivity to insulin of health man. Clin Sci 1935, 2: 67-94.

29. Garg, A., Bonanome, A., Grundy, S., Zhang, Z. \& Unger, R.H. Comparison of a high carbohydrate diet with a high monounsaturated fat diet in patients with non-insulin dependent diabetes mellitus. $N$ Engl $J$ Med 1988, 319: 829-834.

30. Lalor, B.C., Bhatnagar, D., Winocour, P.H. et al. Placebocontrolled trial of the effects of guar gum and metformin on fasting blood glucose and serum lipids in obese, type 2 diabetic patients. Diabetic Med 1990, 7: 242-245.

31. Durrington, P.N. Secondary hyperlipidaemias. In: Hyperlipidaemia Diagnosis and Management. Wright, London, 1989, pp. 219-276.

32. Pietri, A.O., Dunn, F.L., Grundy, S.M. \& Raskin, P. The effect of continuous subcutaneous insulin infusion on verylow-density lipoprotein triglyceride metabolism in type I diabetes mellitus. Diabetes 1983, 32: 75-81.

33. Winocour, P.H., Durrington, P.N., Hunt, L., Anderson, D.R. \& Cohen, H. Effect of short-term improvements in glycaemic control on serum lipoproteins in insulin-treated diabetics. In: Crepaldi, G., Tienzo, A. \& Baggio, L. (eds), Diabetes, Obesity and Hyperlipidaemia. Excerpta Medica International Congress Series 681 1985, pp. 269-270.

34. Durrington, P.N. Drug therapy of hyperlipidaemia. In Hyperlipidaemia. Diagnosis and Management. Wright, London, 1989, pp. 195-218.

35. Winocour, P.H., Durrington, P.N., Bhatnagar, D. et al. The effect of bezafibrate on very low density lipoprotein, intermediate density lipoprotein and low density lipoprotein composition in type 1 diabetes associated with hypercholesterolaemia or combined hyperlipidaemia. Diabetic Medicine, in press.

36. Alberti, K.G.M.M., Jones, I.R., Laker, M.F., Swai, A.B.M. \& Taylor, R. Effects of bezafibrate on metabolic profiles in non-insulin-dependent diabetes mellitus. $J$ Cardiovasc Pharmacol 1990, 16 (Suppl 9): 521-525. 
37. Breddin, H.K., Krzwanek, H.J., Althoff, P. et al. PARD: platelet aggregation as a risk factor in diabetes: results of a prospective study. In: Janka, H.V., Mehnart, H. \& Standl, E. (eds), Macrovascular Disease in Diabetes Mellitus. Georg Thieme Verlag, Stuttgart, 1985, pp. 63-68.

38. Winocour, P.H., Durrington, P.N., Bhatnagar, D. et al. Double-blind placebo-controlled study of the effects of bezafibrate on blood lipids, lipoproteins and fibrinogen in hyperlipidaemic type 1 (insulin-dependent) diabetes mellitus. Diabetic Med 1990, 7: 736-743.

39. Borch-Johnsen, K. \& Kreiner, S. Proteinuria: value as a predictor of cardiovascular mortality in insulin dependent diabetes mellitus. $\mathrm{Br} \mathrm{Med} J$ 1987, 294: 1651-1654.

40. Winocour, P.H., Durrington, P.N., Ishola, M., Anderson, D.C. \& Cohen, K. Influence of proteinuria on vascular disease, blood pressure, and lipoproteins in insulin-dependent diabetes mellitus. $\mathrm{Br}$ Med J 1987, 294: 1648-1651.

41. Seviour, P.W., Teal, T.K., Richmond, W.R. \& Elkeles, R.S Serum lipids and macrovascular disease in non-insulin dependent diabetics: a possible approach to prevention. Diabetic Med 1988, 5: 166-171.

42. Goldberg, R., La Belle, P., Zupkis, R. \& Ronca, P. Comparison of the effects of Lovastatin and Gemfibrozil on lipids and glucose control in non-insulin dependent diabetes mellitus. Am J Cardiol 1990, 66: 16B-21B.

43. Carlson, L.A., Olsson, A.G. \& Ballantyne, D. On the rise in low density and high density lipoproteins in response to the treatment of hypertriglyeridaemia in type IV as V hyperlipoproteinaemias. Atherosclerosis 1977, 26: 603-609.

44. Garg, A. \& Grundy, S. Lovastatin for lowering cholesterol levels in non-insulin dependent diabetes mellitus. $N$ Engl $J$ Med 1988, 318: 81-86.

45. Yoshino, G., Kazumi, T., Iwai, M. et al. Long form treatment of hypercholesterolaemic non-insulin dependent diabetes with pravastatin (CS 514). Atherosclerosis 1989, 75: 67-72.
46. Rabelink, A.J., Hene, R.J., Erkelens, D.W., Joles, J.A. \& Koomans, H.A. Effects of simvastatin and cholestyramine on lipoprotein profile in hyperlipidaemia of nephrotic syndrome. Lancet 1988, ii: 1335-1338.

47. Golper, T.A., Illingworth, D.R., Morris, C.D. \& Bennett, W.M. Lovastatin in the treatment of multifactonal hyperlipidaemia associated with proteinuria. Am J Kidney Dis 1989, 13: 312-320.

48. Vaga, G.L. \& Grundy, S.M. Lovastatin therapy in nephrotic hyperlipidaemia. Effects on lipoprotein metabolism Kidney Int 1988, 33: 1160-1168.

49. Crouse, J.R. Hypertriglyceridaemia: a contraindication to the use of bile acid binding resins. Am J Med 1987, 83: 243-248.

50. Fulcher, G.R., Jones, I.R. \& Alberti, K.G.M.M. Improvement in glucose tolerance by reduction of lipid concentrations in non-insulin-dependent diabetes mellitus. Diabetes News 1988, 9: 4-6.

51. Kasim, S.E., Stern, B., Khilnani, S. et al. Effects of omega-3 fish oils on lipid metabolism, glycemic control, and blood pressure in type I diabetic patients. J Clin Endocrinol Metab 1988, 67: 1-5.

52. Glauber, H., Wallace, P., Griver, K. \& Brechtel, G. Adverse metabolic effect of omega-3 fatty acids in non-insulindependent diabetes mellitus. Ann Intern Med 1988, 108: 663-668.

53. Borkman, M., Chisholm, D.J. \& Furler, S.M. Effects of fish oil supplementation on glucose and lipid metabolism in NIDDM. Diabetes 1989, 38: 1314-1319.

54. Durrington, P.N. \& Miller, J.P. Double-blind, placebocontrolled, cross-over trial of probucol in heteozygous familial hypercholesterolaemia. Atherosclerosis 1985, 55: 187-194. 